\title{
INELASTIC ELECTRON SCATTERING IN DOUBLE-BARRIER RESONANT TUNNELING STRUCTURE REVEALED WITH PHOTOEXCITATION
}

\author{
T. Figielski, A. Mąkosa, T. Wosiński \\ Institute of Physics, Polish Academy of Sciences \\ Al. Lotników 32/46, 02-668 Warszawa, Poland \\ P.C. Harness and K.E. Singer \\ Department of Electrical Engineering and Electronics \\ University of Manchester Institute of Science and Technology (UMIST) \\ PO Box 88, Manchester, M60 1QP, U.K.
}

We investigated current-voltage and photocurrent-voltage characteristics of a double-barrier resonant tunneling structure based on AlGaAs. To explain the observed "double-step" feature of the characteristics, we have proposed a mechanism including a multiple phonon emission of an electron dwelling in the quantum well.

PACS numbers: $73.40 . \mathrm{Gk}, 85.30 . \mathrm{Mn}$

A double-barrier resonant tunneling structure (DBRTS) grown by molecular beam epitaxy at UMIST [1] was investigated. It consisted of two 8-nm $\mathrm{Al}_{0.4} \mathrm{Ga}_{0.6} \mathrm{As}$ barriers separated by a 4-nm GaAs well and had incorporated in the emitter region an $n$-doped three-period superlattice. Measurements were performed on mesa diodes of the area $(50 \mu \mathrm{m})^{2}$ fabricated from such a structure. A $5 \Omega$ resistor was connected in parallel with the device in a close proximity to the investigated structure.

We measured current-voltage $I(V)$ and photocurrent-voltage characteristics of the diodes in the temperature range $80-300 \mathrm{~K}$. The photocurrent was excited by overall illumination of the structure with a monochromatic light $(\lambda \approx 750 \mathrm{~nm})$ chopped at a low frequency.

Figure 1a shows a part of the $I(V)$ characteristic at $80 \mathrm{~K}$. A "double-step" feature exhibiting a broad shoulder behind the resonance peak is seen in this figure. Such a feature has been commonly observed in characteristics of DBRTS's [2, 3], but its origin has not been satisfatorily explained until now. Foster et al. [4] have argued that it results from time-averaged current oscillations generated in the negative-differential-resistivity region of a diode. 

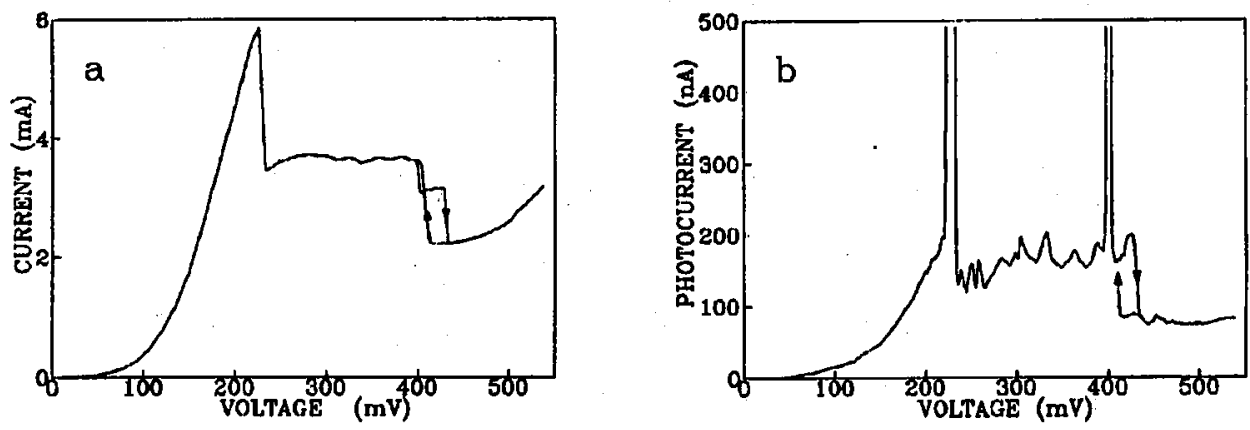

Fig. 1. (a) Current-voltage characteristic of DBRTS diode at $80 \mathrm{~K}$. The slope of the steps at about $0.22 \mathrm{~V}$ and $0.4 \mathrm{~V}$ is determined by the $5 \Omega$ resistor. (b) Photocurrent-voltage characteristic of the same diode. Results for the return voltage run are here omitted for a clarity of the picture.

The most spectacular feature of the diode photocurrent is its giant spike-like enhancement at the bias voltages corresponding to each step. The photocurrent, or at least a dominant part of it, is likely a secondary effect caused by a separation of photo-generated electron-hole pairs at the barriers. Such electrons or holes, entering the quantum well from adjacent emitter or collector regions via tunneling through appropriate barrier, carry into the well an additional electric charge. As a result, the proportion between the voltage drop across the emitter and collector barriers are changed by illumination, which in turn changes the tunneling current through the structure. This is, in fact, a kind of differentiation of the "dark" current flowing through the diode.

An unexpected feature discovered in the photocurrent has been the appearance of some oscillatory structure within the range between the two spikes. An example of such a structure is seen in Fig. 1b, where one can recognize several maxima that correlate with a weak structure noticeable in the "dark" $I(V)$ characteristic. It is worth noting that traces of similar structure could be revealed also in "double-step" features reported in the literature $[2,3]$. This structure is undoubtely an intrinsic property of the diode as the number of observed maxima is independent of the external circuit. Similar structure is also observed in $\mathrm{d} I(V) / \mathrm{d} V$ characteristics of the diode. Since these maxima usually displayed a period close to the longitudal optical (LO) phonon energy in GaAs, this encouraged us to propose an alternative phonon-involved explanation for this feature.

Principal for this explanation is the time spent by an electron in the quantum well where it bounces back and forth between the two barriers before it leaks out through the collector barrier. This time estimated for the diode under resonance conditions is of the order of a few picoseconds. While dwelling in the well, the electron suffers from inelastic scattering mainly owing to the emission of LO phonons. In each of the scattering event an electron wave loses its phase coherence. Phonon emission of hot electrons confined in a well has been thoroughly investigated in 
the literature and hence we know that in GaAs well an average time between two successive emission events is about $0.1 \mathrm{ps}$ and only slightly depends on the electron energy [5]. So, an electron injected into the well can emit some 10 phonons before it escapes from the well.

Further, for simplicity, we will consider a one-dimensional case. When the energy of an incident electron in the emitter region coincides with that of the quasi-bound state of the quantum well, then the electron injected into the well contributes to the coherent resonant tunneling until emission of the first phonon. During this time it gives rise to an enhanced resonant transmission.

Suppose now that the energy of an incident electron exceeds that of the quasi-bound state by a quantity $n \hbar \omega$, where $\hbar \omega$ is the phonon energy, and $n$ is a number no larger than the maximal number of phonons that could be emitted while the electron dwelt in the well. In that case, electron is injected into a scattering (unbound) state in the well where it contributes to a non-resonant tunneling. But after emission of $n$ phonons this electron will attain the quasi-bound state of the well where its wave function forms a standing wave. Although this wave has already lost the memory of its initial phase, it can regain it by interacting with an incoming wave. Thus, such an electron will also contribute to an enhanced resonant transmission.

We believe that the broad shoulder observed behind the main resonance peak consists, in fact, of several additional resonance peaks, whose number equals to the number of phonons emitted during electron dwell in the well. In reality, the situation is more complicated due to an electrostatic feedback between the tunneling current and the voltage drop across the emitter barrier resulting from a space-charge buildup in the well. It causes, first, that a single resonant peak becomes leaned to the right (to higher bias). Eventually this gives rise to intrinsic bistability where the current becomes triple valued at certain applied voltages [6]. Secondly, it means that the voltage drop across the emitter barrier needs not to be proportional to the externally applied voltage, and this makes difficult any qualitative interpretation of the results.

This work is supported by the grant No. 204619101 of the Committee for Scientific Research.

\section{References}

[1] P.C. Harness, R.E. Pritchard, B. Khamsehpour, W.S. Truscott, K.E. Singer, J. Appl. Phys. 71, 3019 (1992).

[2] V.J. Goldman, D.C. Tsui, J.E. Cunningham, Phys. Rev. B 35, 9387 (1987).

[3] S. Charbonneau, J.F. Young, A.J.S. Thorpe, Appl. Phys. Lett. 57, 264 (1990).

[4] T.J. Foster, M.L. Leadbeater, L. Eaves, M. Henini, O.H. Hughes, C.A. Payling, F.W. Sheard, P.E. Simmonds, G.A. Toombs, Phys. Rev. B 39, 6205 (1989).

[5] E. Molinari, C. Bungaro, M. Gulia, P. Lugli, H. Rücker, Semicond. Sci. Technol. 7, B67 (1992).

[6] F.W. Sheard, G.A. Toombs, Appl. Phys. Lett. 52, 1228 (1988). 